\title{
Propiedad intelectual en el Tratado de Libre Comercio con los Estados Unidos ${ }^{1}$
}

\author{
Tatiana Olaya Steevens \\ Estudiante $5^{\circ}$ semestre Contaduría Pública \\ Universidad Santo Tomás \\ Marcela Higuera Triviño \\ Estudiante $5^{\circ}$ semestre Contaduría Pública \\ Universidad Santo Tomás
}

\section{Resumen}

Ante la necesidad de conocer apartes de lo que será el Tratado de Libre Comercio entre Colombia y Estados Unidos, es necesario hacernos participes en temas que podrían afectarnos. Tal es el caso de la propiedad intelectual, uno de los de mayor contrariedad y discusión. Sin embargo, para ver cómo los contadores debemos involucrarnos en este tema, se dará un enfoque centrado en la contabilidad del conocimiento, la cual busca conciliar las necesidades y las dificultades que se forman en el manejo de una organización con el uso y la generación de éste, específicamente en el capital intelectual.

\section{Palabras clave}

Contabilidad del conocimiento; Capital intelectual; Propiedad intelectual; Derechos de propiedad intelectual; Tratado de Libre Comercio; Marcas; Patentes; Medicamentos.

\footnotetext{
${ }^{1}$ Ponencia presentada en el I Congreso de Estudiantes de Contaduría Pública de la Universidad Santo Tomás, octubre de 2006.
} 


\section{Entorno global}

\section{Introducción}

Actualmente cada vez es mayor la integración de las economías nacionales a través del intercambio de flujos financieros y de productos dando a lugar la palabra de moda: "globalización", este concepto hace referencia a la integración de los diversos mercados financieros domésticos en un único mercado financiero internacional, lo que permite a las instituciones de cualquier país que busquen fondos, y no limitarse a localizarlos en su propio mercado.

La globalización económica como proceso histórico, nace como resultado de la innovación humana y el progreso tecnológico, y con esto nos referimos a la creciente integración de las economías mundiales; y a partir de ello nacen algunas circunstancias en las que desplazan a las personas y se facilita la transferencia de conocimientos a través de las fronteras internacionales, gracias a recursos como la tecnología.

A partir de esta realidad, es necesario evaluarnos como profesionales de la contaduría en cuanto a nuestro compromiso y participación de este proceso mundial, el cual nos presenta un entorno competitivo, donde prevalece el conocimiento y la habilidad para desenvolverse, puesto que hoy día el conocimiento juega un papel importante en la organización representado en el capital intelectual y su correcto manejo.

Este fenómeno mundial genera a su vez a las organizaciones, la necesidad de prepararse para enfrentarse a nuevos y más calificados mercados donde como lo mencionamos, la competencia es mayor siendo este factor sinónimo de mayor 
productividad, representando mayores retos para las empresas que desean mantenerse y ampliar sus oportunidades de negocio.

Teniendo en cuenta los requerimientos del mercado y de las empresas, cabe resaltar la necesidad de las organizaciones por mejorar su desempeño, preparación y desarrollo en los nuevos ambientes cada vez más competitivos, donde el contador público conservará y desarrollará su propio campo de acción en la labor de fortalecer a las entidades en su administración, finanzas y control de las operaciones, apoyando con su capacidad profesional el crecimiento de las mismas para que puedan hacer frente a los nuevos retos que les depara el futuro.

Teniendo como base ésta pequeña introducción y además con la llegada del tratado de libre comercio entre Colombia y Estados Unidos es necesario por simple cultura general, y sobretodo por ejercer nuestro derecho como colombianos conocer temas tan importantes que nos han de afectar, los puntos que en el tratan ambos gobiernos como es la propiedad intelectual, uno de los temas más discutidos por el efecto que tendría en el momento que este negocio se haya de cerrar.

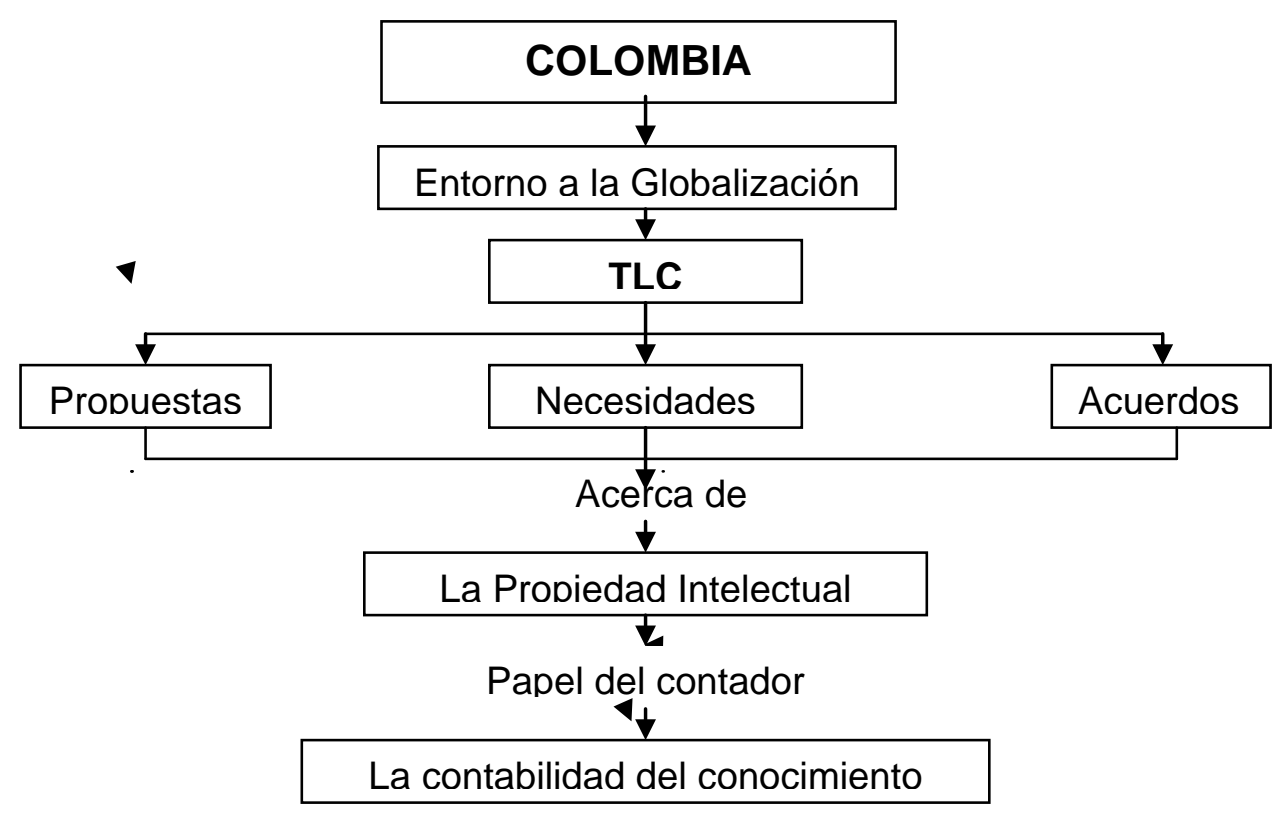




\section{Colombia y el TLC}

\section{Proceso, negociación y resultados}

Para entrar a detallar la situación colombiana en el Tratado de Libre Comercio con EUA, es de gran importancia conocer en que consisten los derechos de propiedad intelectual, los cuales se pueden definir como aquellos que se confieren a las personas sobre las creaciones de su mente. Suelen dar al creador derechos exclusivos sobre la utilización de su obra por un plazo determinado; en el capítulo de propiedad intelectual de dicho acuerdo, se ocupa de una larga lista de temas, de los cuales procuraremos por tratar solo los más importantes, y los cuales a su vez reflejan los debates contemporáneos sobre el alcance, profundidad y exigibilidad de dichos derechos.

Teniendo en cuenta la definición anterior, y la actual tendencia de la economía colombiana la cual se encuentra en una fase de transición e incursión en la segunda fase de la globalización de mercados, centrada o enfocada hacia el comercio internacional, y que mejor muestra de ello con el TLC.

Respecto al tema que nos compete, el TLC nos demuestra que sus intenciones y esfuerzos cada vez presionan más a nuestro país en cuanto al tema de protección de los derechos de propiedad intelectual principalmente de sus empresas y es por eso que Colombia debe reconocer claramente dicha situación.

La negociación del TLC, con Estados Unidos y la Comunidad Andina (CAN), que reúne a Perú, Ecuador y Colombia, quienes especialmente en el tema de propiedad intelectual han trabajado mancomunadamente con el fin último de mantener las normas comunitarias en propiedad industrial, establecida por esta comunidad, a la vez demostrando desarrollos legales de mayor amplitud y sofisticación en materia de propiedad industrial, derechos autor y conexos; sin 
embargo cabe resaltar que los esfuerzos especialmente de la mesa de negociación de la ronda 7 del TLC, se enfocaron hacia temas como la protección de datos, la salud pública, la biodiversidad y los conocimientos ancestrales.

\section{Posición colombiana}

Teniendo en cuenta lo anteriormente mencionado, podemos ver que la posición colombiana se ve enmarcada por el ánimo de conservar y preservar en lo posible la integridad de la norma manejada; también, procurar no exceder las obligaciones contraídas con en el Acuerdo de aspectos sobre los derechos de propiedad intelectual (ADPIC), promulgados por la OMC (Organización Mundial del Comercio) en donde dicho Acuerdo y sus miembros tienen por objeto, entre otras cosas, reducir las distorsiones del comercio internacional y los obstáculos a ese comercio, fomentar una protección eficaz y adecuada de los derechos de propiedad intelectual y asegurarse de que las medidas y procedimientos destinados a hacer respetar dichos derechos no se conviertan a su vez en obstáculos al comercio legítimo; aquí cabe demostrar el interés de EE.UU. al solicitar que los países andinos se adhieran a diez tratados internacionales de Propiedad Intelectual administrados por la OMPI (Organización Mundial de la Propiedad Intelectual, organismo especializado del sistema de organizaciones de las Naciones Unidas que tiene por objetivo desarrollar un sistema de propiedad intelectual internacional, que sea equilibrado y accesible y recompense la creatividad, estimule la innovación y contribuya al desarrollo económico, salvaguardando a la vez el interés público; establecida en 1967 con el mandato de estados miembros para fomentar la protección de la propiedad intelectual en todo el mundo mediante la cooperación de organizaciones nacionales e internacionales), en dos de ellos se pide un compromiso de mejor esfuerzo para lograr la adhesión; Colombia ya es parte de tres de los tratados solicitados (el 
Tratado de Cooperación de Patentes y los dos Tratados de la OMPI de 1996 o tratados Internet) por tanto el debate se concentra en los siete restantes: la Convención de Brúcelas sobre Señales Satelitales, el Protocolo de Madrid sobre Registro Internacional de Marcas, el Tratado de Budapest (Depósito de Microorganismos), la Convención UPOV 91, el Tratado de Derecho de Marcas, (TLT), el Tratado de Derecho de Patentes (PLT) y el Acuerdo de la Haya (Registro Internacional de Diseños Industriales); respecto a esto los países andinos han reaccionado positivamente a algunos de estos tratados (por ejemplo Budapest, que ya figura en nuestra norma andina o al de Brúcelas), pero la aceptación de los restantes depende no solo de la sensibilidad colombiana ante la adopción de compromisos internacionales sino además del balance final del capítulo de Propiedad Intelectual.

De acuerdo con lo mencionado anteriormente, vemos que Colombia como tal goza de un régimen robusto y balanceado en propiedad intelectual y que siendo éste un asunto estratégico para Estados Unidos. Pero al ver esta situación cabe hacernos una pregunta, ¿Cuál es la necesidad de EE.UU. al presionar la utilización dichas normas?, lógicamente sus esfuerzos enfocados hacia la dirección de nuestra legislación más en línea con su propia normativa e intereses particulares; sin embargo el país desde tiempo atrás ha venido negociando asuntos de propiedad intelectual en incontables foros internacionales como la Organización Mundial de Comercio (OMC), la Comunidad Andina (CAN), la Organización Mundial de la Propiedad Intelectual (OMPI), el ALCA e incluso otros TLC como el negociado con México hace más de una década (el TLC G-3). ${ }^{2}$

\footnotetext{
${ }^{2}$ Tomado "LA PROPIEDAD INTELECTUAL EN EL TLC" en: www.usergioarboleda.edu.co/tlc/propiedad intelectual tlc mirada.htm
} 


\section{Legislaciones propiedad intelectual - Colombia}

En Colombia las creaciones intelectuales que son objeto de propiedad intelectual versan sobre dos concepciones claramente marcadas, como son, las referentes a la estética, es decir a obras literarias, artísticas y demás, que específicamente corresponden a los Derechos de Autor; por otro lado encontramos las referentes a las actividades industriales tales como, marcas y patentes, denominadas propiedad industrial. Partiendo de estas dos concepciones podemos ver la normatividad colombiana frente al tema de la Propiedad Intelectual.

¥ DERECHOS DE AUTOR: La protección de dichos derechos esta regulada por la ley 23 de 1982, la Ley 44 de 1993 y la Decisión 351 del Acuerdo de Cartagena y sus decretos reglamentarios.

El objeto de estas normas se centra en proteger las obras artísticas, científicas y literarias que pueden ser reproducidas o divulgadas de cualquier forma, así como amparar los derechos de los artistas, intérpretes, productores de fonogramas y titulares de programas de computador (software).

La protección de los trabajos artísticos y literarios no depende de su registro, y por lo tanto la omisión de éste no es obstáculo para que goce de salvaguarda, ya que la titularidad de la obra se obtiene con la creación de la misma, más no con su registro. Sin embargo, se recomienda el registro de la obra ante la Dirección Nacional de Derechos de Autor, de manera que pueda oponerse como defensa frente a las reproducciones no autorizadas, ya que constituye un eficaz medio de prueba del derecho que facilita su negociación y defensa judicial. 
- PROPIEDAD INDUSTRIAL: El régimen común sobre Propiedad industrial es la Decisión 486 de la Comunidad Andina reglamentada en Colombia bajo el Decreto 2591 del 13 de diciembre de 2000 y la Circular Única de la Superintendencia de Industria y Comercio.

La protección de la propiedad industrial se divide en dos grandes temas: signos distintivos y nuevas creaciones. Los signos distintivos comprenden las marcas, los lemas comerciales, los nombres, enseñas comerciales e indicaciones geográficas. Las nuevas creaciones comprenden las patentes de invención, patentes de modelo de utilidad, diseños industriales y esquemas de trazado de circuitos integrados. (Ver Cuadro 1$)^{3}$

\section{Cuadro 1}

Obras artísticas, científicas y literarias que puedan ser reproducidas o divulgadas de cualquier forma.
PROPIEDAD INTELECTUAL - COLOMBIA

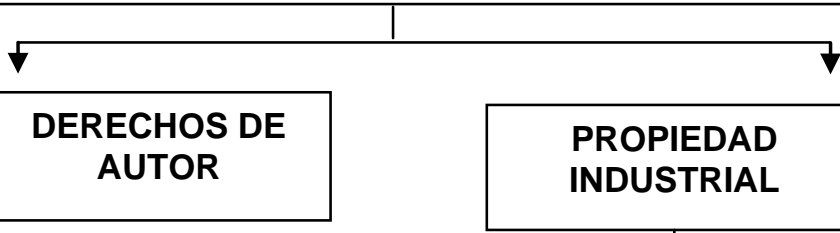

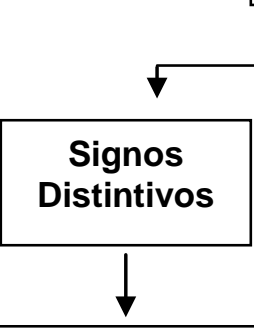

*Marcas

* Lemas comerciales

*Nombres y enseñas comerciales

*Denominaciones de origen (indicaciones geográficas)
*Patente de invención

*Patente de modelo de utilidad

*Diseños Industriales

*Esquema de trazado de circuitos integrados 


\section{Temas de mayor interés en propiedad intelectual en el TLC}

\section{a) Salud pública y medicamentos}

El objetivo de los ministros de salud de los países participantes en este acuerdo bilateral, es proteger el acceso a los medicamentos y reducir impactos desfavorables a la salud pública en razón a que las medicinas son insumos esenciales para la atención de la salud y la protección de la vida. Dentro de los asuntos negociados se destacan ${ }^{4}$ :

1. Compensación por retrasos irrazonables en la oficina de patentes: aplica a cualquier solicitud de patente, cuyo trámite tome más de cinco años en la oficina nacional de patentes (en Colombia, la Superintendencia de Industria y Comercio). Por consiguiente, en aquellas solicitudes de patentes cuyo trámite se prolongue más allá de ese término, el titular de la patente tendrá el derecho a pedir una restauración del término de la patente; compensación en tiempo que se debe sumar al plazo de veinte años hoy vigente. El plazo de la compensación no está definido en el TLC y corresponde al legislador nacional hacerlo.

Es oportuno indicar que los plazos de trámite establecidos por el Régimen Comunitario Andino (Decisión 486) imponen que una solicitud de patente debe decidirse en un plazo aproximado de cuarenta y dos meses, mientras que como se vio, el TLC ha puesto un término equivalente a sesenta meses, más allá del cual se considerará la demora un "retraso irrazonable" que debe generar una compensación en tiempo para el titular de la patente.

\footnotetext{
${ }^{3}$ Cuadro 1. Tomado Página Web PROEXPORT COLOMBIA; Marco Legal 2006. Propiedad Intelectual. En: www.proexport.xom.xo/VbeContent/Library/documents/DocNewsNo2726DocumentsNo5547.PDF

${ }^{4}$ Aspectos Negociados, tomados de artículo "La salud pública y la discusión de Propiedad Intelectual en el TLC Colombia-Estados Unidos" Originalmente página Web en:

www.elsignovital.com.co
} 
Respecto a este tema, podemos ver la intención estadounidense por buscar la ampliación del tiempo de las patentes lo cual está representado en el mayor aprovechamiento y aumento del monopolio por parte de las multinacionales farmacéuticas.

2. Compensación por reducción irrazonable en el plazo efectivo de la patente en el trámite sanitario: a diferencia del caso anterior, éste aplica únicamente a solicitudes de patentes del sector productos farmacéuticos. El lenguaje un poco enrevesado se refiere a los derechos derivados de la patente de un producto farmacéutico - patentado en Colombia-, que siendo comercializado por vez primera en el país, se vean -tales derechosmenoscabados por demoras en la oficina sanitaria (en nuestro caso, el INVIMA). De manera similar al primer caso, nuestro legislador debe definir el tiempo de compensación y además (a diferencia) determinar que se entiende por demora, es decir, a partir de cuando ocurre el retraso en la oficina sanitaria.

3. Protección de datos de prueba: los datos de prueba son los estudios de seguridad y eficacia que se entregan a la autoridad reguladora para obtener la aprobación de comercialización de nuevos productos farmacéuticos o agroquímicos. En Colombia se protegen estos estudios mediante los decretos 2085/02 y 502/03, respectivamente. Lo pactado en el TLC en productos farmacéuticos sigue de cerca a la legislación nacional. Es decir, se protegen los datos de prueba por cinco años (5) contados a partir de la aprobación del registro sanitario. En ese periodo, nadie distinto a quien haya entregado esos estudios a la autoridad sanitaria -generalmente la compañía que desarrolló el medicamento- puede hacer uso directo o indirecto de los mismos para obtener un permiso de comercialización. En otras palabras, un productor de medicamentos genéricos -que no ha 
realizado los estudios de seguridad y eficacia y que de manera general no tiene acceso a sus resultados- debe esperar el vencimiento del plazo correspondiente para hacer uso de dichos estudios a fin de obtener su propio registro sanitario sobre el medicamento de que se trate.

4. Asuntos nuevos en protección de datos de prueba: En carta adjunta al capítulo de propiedad intelectual se aclara que la protección de datos de prueba no impedirá que los países hagan uso del mecanismo de flexibilización negociado en el marco de la Organización Mundial del Comercio sobre licencias obligatorias de patentes. De otro lado, se evidencian en el texto del capítulo de propiedad intelectual ciertas novedades en la materia que a buen seguro van a generar controversia en los países involucrados, y que podrían señalarse como adiciones a la legislación vigente en Colombia: la protección a datos de prueba se dará sin importar si éstos son divulgados o no -hoy Colombia solo protege información no divulgada - y la protección de datos de prueba se extenderá a casos de aprobación por referencia del registro sanitario -el decreto 2085/02 no regula la aprobación por referencia, toda vez que en este tipo de aprobaciones no se exige la presentación de los datos de prueba -.

Finalmente, en lo que ha sido anunciado como un avance, se pactó un plazo de cinco años, dentro de los cuales el titular de los datos de prueba debe solicitar la protección de los mismos en el país, so pena de perder esa protección. Ese plazo se cuenta a partir de la primera aprobación en el país extranjero y solo aplica para casos de aprobación por referencia. Que ocurre cuando el solicitante no hace uso del mecanismo de la aprobación por referencia sino de la aprobación ordinaria, seguramente será objeto de una interesante discusión que aún no se ha abierto. 


\section{Vinculación del registro sanitario con la protección de patentes}

(conocido como linkage): las partes en el TLC adquirieron la obligación de evitar que una persona distinta al titular de la patente, "reclame" un producto sanitario -o método de uso- cubierto por esa patente durante el término de vigencia de ésta. De esta manera complementaria, se estableció la obligación para la autoridad sanitaria de informar al titular de la patente de la identidad de quien pretenda obtener un registro sanitario sobre el medicamento objeto de la patente durante su vigencia, siempre y cuando el titular de la patente informe de la existencia de la misma a la autoridad sanitaria. Ansiedad extrema ha causado la primera de las figuras en ciertos sectores de la producción nacional, señalando que nuevamente corresponde al legislador nacional determinar como se implementa esa obligación. Respecto de esta medida, es preciso decir que los medicamentos genéricos siguen y seguirán siendo en la medida de lo posible, la única opción posible para la universalidad del acceso a la salud en Colombia, y el impedimento a los diversos laboratorios que no posean la patente totalmente, para elaborar y comercializar el medicamento genérico de determinadas medicinas.

6. Cláusula Bolar: esta es una excepción al derecho del titular de una patente y permite a un tercero realizar ciertos actos, que le aseguren entrar al mercado de manera inmediata a la expiración de la patente. En otras palabras, el productor de medicamentos genéricos puede realizar las actividades que le permitan conseguir la información necesaria de apoyo a su solicitud de registro sanitario, sin que ello signifique infracción a la patente que eventualmente cubra el medicamento. Se señala que el Régimen Común Andino, Decisión 486, no cuenta con esa figura. Frente a esta cláusula lo que busca realmente EE.UU., es que el productor del 
genérico se demore varios meses en ingresar su medicamento, dando paso a que ese tiempo se lo gane el monopolio del innovador.

7. El nombre común y la distintividad de la marca: se ha pactado que cuando un país exija la utilización del "nombre común" -es decir, el nombre del principio activo o nombre genérico- en conjunción con la marca de un producto farmacéutico- esta exigencia no deberá menoscabar el uso o efectividad de la marca. La legislación colombiana determina que en algunos casos -especialmente en medicamentos de interés público- el nombre común puede llegar a ocupar hasta la mitad del espacio destinado a la etiqueta del producto. Esto se hace para orientar al público consumidor y evitarle confusiones en la adquisición del medicamento. Es preciso anotar que en la mesa de negociación se estableció que la exigencia colombiana no afecta lo pactado en el TLC.

Los puntos referidos anteriormente son los puntos clave o asuntos de mayor interés tratados respecto a la salud pública y medicamentos en el país, y en los cuales la negociación se torno más demorada.

Respecto al tema de medicamentos, se ha realizado cualquier cantidad de estudios donde se busca precisar las implicaciones de las nuevas medidas de protección de propiedad intelectual, específicamente el área de fármacos, ejemplo de ello lo es la Academia Nacional de Medicina y la Organización Panamericana de la Salud (OPS $)^{5}$, quienes en el foro realizado el 7 de Julio de 2005, realizaron tres estudios acerca de implicaciones del TLC en la salud de Ios colombianos, realizados respectivamente por IFARMA/OPS-OMS, Universidad Nacional y la Fundación FEDESARROLLO; De acuerdo a los

\footnotetext{
${ }^{5}$ Cifras y resultados tomados página Web Organización Panamericana de la Salud. Articulo "¿El TLC tiene impacto en la salud de los colombianos?" en: www.col.ops-oms.org
} 
diferentes resultados de los estudios muestran que el acuerdo internacional sobre propiedad intelectual ADPIC actualmente ya tiene un efecto importante sobre el costo del tratamiento para las patologías prevalentes en el país y podría generar costos adicionales para los individuos y para el país con un efecto negativo en el acceso a medicamentos exclusivos, o de monopolio.

El efecto sobre el acceso de la población colombiana a los medicamentos, si no es compensado por un aumento correspondiente de los recursos financieros del sector, podría afectar hasta un $30 \%$ de la población actualmente cubierta por el sistema de aseguramiento en un futuro no muy lejano.

Un ejemplo claro de las implicaciones del TLC en la salud del colombiano, se demuestra en el estudio IFARMA/OPS donde se evidencia que podría darse un encarecimiento de los fármacos antirretrovirales para tratar los pacientes con VIH/SIDA, que podría determinar ausencia de acceso para 3000 pacientes con la enfermedad, lo cual denota un peligro inminente.

Nos parece de gran importancia referirnos a una sugerencia realizada por la OMS la cual indica que para que se lleve a cabo es importante que productores, decisores públicos, médicos y consumidores puedan tener acceso a la misma calidad de la información sobre medicamentos para que con ello, el diálogo y las decisiones puedan darse en condiciones de igualdad y así mismo reflejar el bien común a todos los que en dichas decisiones participan.

Teniendo en cuenta las implicaciones y disposiciones supuestamente pactadas en el TLC con Estados Unidos es de vital importancia la preparación que Colombia disponga para llevar a cabo con éxito dicho acuerdo bilateral, dentro de las cuales, -basadas en el estudio realizado por la Academia Nacional de 
Medicina y la Organización Panamericana de la Salud (OPS ) mencionado anteriormente- tenemos:

- Uso de sólidas herramientas para el monitoreo del impacto de la propiedad intelectual en su evolución presente y futura.

- Información científica accesible a los sujetos del estado y sociales.

- Fortalecimiento de las entidades nacionales con función de control de la calidad

- Atención a la equidad en el acceso de la población a salud y a sus derechos sociales para evitar que como efecto de negociaciones de carácter comercial se vea afectada una porción importante de la población, con pérdida del acceso a medicamentos.

\section{b) Biodiversidad}

De acuerdo con este tema de carácter importante, sobre todo en países tercer mundistas como nosotros, puesto que la más rica biodiversidad () la encontramos en ellos; Los derechos de propiedad intelectual se hallan atados al aprovechamiento sustentable de la biodiversidad en los países y las comunidades que la poseen y las trasnacionales pretenden extender las patentes a los seres vivos (animales y plantas), mientras el Tercer Mundo, poseedor de los recursos, intenta contener esta pretensión que juzgan una amenaza a la soberanía, la tradición y el sustento de las comunidades locales. La biodiversidad es estratégica para las pretensiones de las farmacéuticas, puesto que para dichas 
multinacionales es de vital importancia el acceso a plantas y animales, de las cuales puedan sacar cualquier tipo de beneficio.

Al principio de las negociaciones EE.UU., por medio del proyecto de tratado de libre comercio (TLC), que entregó la Oficina del Representante Comercial de Estados Unidos, Robert Zoellick, menciono en el artículo 8: «Cada parte (cada país que firme el TLC) deberá permitir las patentes para las siguientes invenciones: a) plantas y animales, y b) procedimientos diagnósticos, terapéutico y quirúrgicos para el tratamiento de humanos y animales» (Gómez Carvajal, 2004); sin embargo dicha "iniciativa norteamericana" no fue exitosamente aceptada por Colombia lo cual nos da alivio pues se hubiera puesto en peligro nuestra riqueza natural y biodiversidad.

Podemos ver como la posición colombiana se torno un poco flexible en un principio (aunque se opuso al cierre de las negociaciones), en cuanto a este tema teniendo en cuenta su magnitud, pues la riqueza colombiana representada en recursos naturales, principalmente plantas y animales, a los cuales tendrían acceso sin mayores restricciones, viéndonos afectados en cuanto a nuestra reserva natural y cultural tan extensa y solicitada por otras naciones, para su propio beneficio comercial como en el caso de los medicamentos y demás permitiéndole que se hagan dueños de los descubrimientos de las comunidades indígenas que son quienes experimentan constantemente con la naturaleza, pero que no cuentan con los recursos para patentar y legalizar sus descubrimientos y no ven la necesidad de hacerlo porque de hecho la creación la realizan en su territorio.

Una de las múltiples divergencias entre lo países andinos y EE.UU. se centra en que éste último se ha negado a reconocer que patentes basadas en material genético extranjero, tengan como requisito el consentimiento previo e informado 
del país de origen del material y vemos como en posición absolutamente contraria, los países andinos no solo reivindican esa obligación, sino que además consideran prioritario que tal mandato se incluya en el TLC, lo cual hasta el momento no es claro si fue incluido en los textos firmados en las negociaciones, debido a la falta de información al público de los textos originales negociados.

\section{Cierre de negociaciones}

El 17 de febrero de 2006, la mesa de negociación correspondiente a la propiedad intelectual quedo cerrada, donde el gobierno después de un largo tiempo trato de hallar un acuerdo justo para el país.

Al cierre de esta mesa, hubo varios puntos destacados:

1. Mantener la capacidad de tomar medidas necesarias para la defensa de la salud pública, incluyendo los instrumentos como licencias obligatorias y las importaciones paralelas.

2. Conservación del estándar actual de protección en materia de datos de prueba y patentes. Colombia seguirá sin otorgar patentes sobre usos ó sobre segundos usos, y métodos terapéuticos, de diagnóstico y quirúrgicos.

3. Colombia preserva la independencia funcional de autoridad sanitaria.

4. El tratado con los países andinos, es el primero que incluye normas para protección de biodiversidad y para evitar actos de biopiratería, por otro lado es el primero que incluye normas para la promoción de la innovación y desarrollo tecnológico en el marco de transferencia de tecnología.

Esta información fue poco de lo oficial que pudimos recolectar dentro de nuestra investigación, puesto que encontramos la dificultad para poder acceder libremente a los textos del TLC, acordados en el cierre de la negociación. 
Para entender mejor estos efectos y el papel que desempeñarían los contadores es necesario iniciar con una breve descripción de lo que es la propiedad intelectual y la contabilidad del conocimiento.

\section{Contabilidad del conocimiento}

La contabilidad del conocimiento "surge como respuesta a las problemáticas derivadas de la administración del capital intelectual" (Mantilla, 2004, 35 y ss.); para desarrollar el concepto de contabilidad del conocimiento es necesario conocer otras definiciones como son la economía del conocimiento, la administración del conocimiento, entre otras para poder desarrollar el concepto de capital intelectual y llegar finalmente a enfatizar en el tema discutido en el TLC como es la propiedad intelectual.

La economía del conocimiento se desarrolla a raíz del hecho de ser hoy en día de mayor importancia el conocimiento (Recurso Humano) que los recipientes del mismo (sistemas expertos, bases de datos...). Se ha convertido en una necesidad, y también en parte para medir la competitividad comercial y en general económica de un ente, la valoración de los activos intangibles, como el conocimiento, marcas y patentes. Se puede ver el desarrollo desde 1998 donde se ha realizado el diseño y formulación del estándar internacional de contabilidad NIC 38-(IAS 38) donde se nombran unos aspectos básicos para la valorización y revelación en los estados financieros. Otra de las innovaciones contables del conocimiento es el "Study 7" (IFAC-FMAC), el cual define algunas diferencias en relación con dicho asunto, debido a que determina la dirección de los nuevos procesos de contabilidad moderna, los cuales están siendo considerados más convenientes como instrumentos de información para la gerencia del capital intelectual humano (Díaz Inchicaque, 2003). 
Se genera entonces, con esas definiciones y aspectos unas diferencias relevantes entre lo que es una economía Industrial y una economía del Conocimiento (Mantilla, 2004):

- Abundancia: El conocimiento y la información crecen constantemente mediante la aplicación.

- Des-ubicación: no se necesita operar en ubicaciones físicas dedicadas.

- Des-localización: se escapan de barreras legales.

- Comparabilidad: Productos o servicios con conocimiento generan una mayor rentabilidad.

- Contextualidad: Los precios que se le concedan a este conocimiento dependen del contexto donde se deseen desarrollar, no para todos los usuarios tienen la misma importancia.

- Competencia Humana: Componente clave de la generación de conocimiento.

La Administración del Conocimiento debido al valor que genera el conocimiento debe ser manejada con los procesos esenciales de la organización (Parker Rosell, 2006). La administración del conocimiento es, según Skyme (2005) la administración explícita y sistemática del conocimiento vital y sus procesos asociados de creación, recolección, organización, difusión, uso y explotación. Requiere trasformar el conocimiento personal en conocimiento útil para la organización.

Es tal la importancia del conocimiento que se han generado una serie de discusiones sobre el manejo y administración de este, su importancia se ve reflejada en la salida de nuevas normas internacionales que buscan de forma apropiada administrar el manejo del conocimiento, pero también una serie de contradicciones en cuanto a su creación ya que se debe tener cuidado en no llegar 
a cosificar a las personas generadoras de conocimiento. En el desarrollo de este nada es terminante, ya que siempre se encontrara la necesidad de continuar investigando, y es por esto que se afirma entonces que el conocimiento se encuentra en constante surgimiento ya que no existe nada absoluto en que se pueda regir el desarrollo económico u organizacional.

De acuerdo con Mantilla (2004) debido a lo cambiante del conocimiento se puede clasificar en diferentes tipos como son:

- Conocimiento como hecho, similar a la información.

- Conocimiento como habilidad, con una importante dimensión humana.

El Capital Intelectual se ve mejor definido en el Study 7, constituido por:

- Capital Humano: que está en la mente de los individuos: conocimiento, competencias, experiencias, saber-cómo.

- Capital Organizacional (estructural): el que permanece luego que los empleados regresan de su casa: procesos, sistemas de información, bases de datos, etc.

Algunos añaden otro elemento debido a la importancia y reconocimiento que tiene:

- Propiedad Intelectual: marcas registradas, copyright, licencias, patentes, etc.

En Colombia se tiene un problema en cuanto a la medición de este tipo de capital, en el decreto 2649/93, capitulo III, normas básicas de contabilidad, se describe el concepto de valuación o medición donde se incluyen conceptos como el de valor histórico, valor actual, valor de realización o de mercado y valor presente, como un 
forma de medir la contabilidad y reflejar lo mejor posible las operaciones del ente económico (cuya definición también es tratada en este capitulo); sin embargo, aunque refleja la posibilidad de cuantificar por valor de mercado los beneficios o sacrificios que generan activos como los intangibles, también se refiere luego a la prudencia como una necesidad de no subestimar los activos, aceptando al valor de realización o de mercado como un criterio de medición más no como un indicador del desempeño económico en la organización. Finalmente en Colombia lo que se viene trabajando desde hace mucho tiempo es una contabilidad por valor histórico, práctica que se ve en desacuerdo con lo estipulado en los estándares internacionales de contabilidad.

Entonces se estipula realmente el problema en la forma como se quiere valorar el conocimiento, por lo que se han ido elaborando formas de hacerlo como son el Balanced Scorecard, EVA, MVA, SVA, circulo aprendizaje-conocimiento-valor, auditoria de procesos de negocios, y KVA (Mantilla, 2004).

1. Balanced Scorecard: creado por Robert Kaplan y David Norton, en un estudio realizado en 1990. Este se realizó por la necesidad de buscar nuevas formas de medir el desempeño organizacional. Es usado como una medida de información y aprendizaje, más no como un sistema de control.

Se desarrolló bajo la perspectiva: financiera, cliente, interna e innovación y aprendizaje. El nombre refleja el balance entre objetivos de corto y largo plazo, entre medidas financieras y no financieras, entre indicadores de atraso y de liderazgo y entre perspectivas externas e internas de desempeño.

- Perspectiva de procesos o interna busca mejoran los procesos de la empresa en sentido del tiempo y la innovación. 
- Perspectiva de Aprendizaje/crecimiento: capacidad para acumular la experiencia y mejorar. Encontramos un desarrollo de la personas.

- Perspectiva del cliente: Tener en cuenta que este la razón de la empresa.

- Perspectiva financiera: Como obtener buena rentabilidad. Utilidades.

El Balanced Sacorecard es una medición crítica del desempeño gerencial y de negocios, mirándolo en un conjunto más integrado de mediciones que vincula el desempeño del cliente actual, procesos internos, empleados y sistemas hacia el éxito financiero en largo plazo.

2. Modelo de valoración de negocios: parte del hecho de la necesidad de ir más allá de los números tradicionales y ayudar a implementar la estrategia. Es el modelo para valorar la planeación de una empresa, estructurando tres niveles:

a. Cualidad de liderazgo = Administración.

b. Creación de Valor = Operaciones + Desempeño.

c. Generación de ingresos = Negocio central + Mercado + Competencia.

\section{Valor Económico Agregado (EVA) y Valor de Mercado Agregado} (MVA): Economic Value Added para Stewart (s/f) es una medida de desempeño financiero que se acerca más que cualquier otra para capturar la verdadera ganancia económica de una empresa, tomando la utilidad operacional neta después de impuestos menos un gasto de capital. Si la rentabilidad retorno sobre el capital de una compañía excede sus costos de capital, esta creado verdadero valor a los accionistas. EVA también es la medida de desempeño mas directamente vinculada a la creación de ganancias de los accionistas. Stern Stewart \& Co. guían las compañías a 
través de la implementación completa de la base financiera gerencial-EVA e incentiva un sistema de compensación que da a los gerentes una información superior - y una motivación superior- para tomar decisiones que puedan crear mas ganancias a los accionistas en cualquier propiedad publica o empresa privada.

EVA busca dar explicación a las partidas no-financieras en una empresa para ser sencillamente entendidas y usadas en la administración del negocio sea cual sea.

Ambos indicadores se han ido implementando cada vez más debido a la necesidad que los accionistas e inversionistas tienen de información útil para la toma de sus decisiones y para ver el correcto desempeño que presenta la empresa.

\section{Análisis del Valor de Accionistas (SVA): Shareholder Value Análisis es} una técnica desarrollada por el profesor Alfred Rappaport (Nortwestern University's J.L. Kellong Graduate School of Management) para observar el valor estratégico en una compañía. Equivale a observar el flujo de efectivo antes y después de realizar una planeación, así como sumarle la seguridad comercial y otras inversiones que son no-operacionales restando las deudas de la corporación ${ }^{6}$.

5. Circulo aprendizaje-conocimiento-valor: en la era industrial la medición de la materia prima era lo más importante, en la economía moderna lo realmente importante es la construcción de una organización sólida e innovadora por medio del conocimiento, así que lo que busca el circulo

\footnotetext{
${ }^{6}$ Life Style Extra: finance, news \& Entertainment, en: http://www.Ise.co.uk/financeglossary.asp?searchTerm=\&iArticleID=952\&definition=shareholder_val ue_analysis.
} 
aprendizaje-conocimiento-valor, es darle valor a la información generada y contenida dentro de los productos finales en términos de los procesos que usaron conocimiento. La nueva materia prima es el conocimiento.

La contabilidad del conocimiento busca entonces conciliar las necesidades y las dificultades que se forman en el manejo de una organización con el uso y la generación de conocimiento llamado, capital intelectual, el autor Samuel Alberto Mantilla (2004) hace referencia al respecto: "Mas allá de los activos fijos tradicionales, las organizaciones valoran hoy la consecución de su visión-misiónobjetivos a partir de acciones estratégicas, siendo una de las mas importantes el uso competitivo del recurso crítico denominado conocimiento. Es el capital intelectual la clave. Y la contabilidad del conocimiento el instrumento".

La importancia de reconocer que la contabilidad general y tradicional hoy en día no es suficiente como instrumento para gestionar, dirigir y evaluar la empresa radica, entre otras cosas, en que las organizaciones ya se dieron cuenta de la necesidad de medir los demás activos que en ella se encuentran y afectan como es el conocimiento, que comprende cuatro procesos básicos: El capital intelectual (conocimiento como activo); administración de la información (conocimiento como información); participación del conocimiento (conocimiento como actividad); y aprendizaje (conocimiento como gente).

Para medir el capital intelectual se ha usado la formula de la diferencia entre el valor del mercado y el valor en libros que ha ayudado a reconocer factores que normalmente no se reconocen en el curso normal de la realización de la contabilidad. 
El capital intelectual está conformado por varios componentes de los que más se destaca la propiedad intelectual ya que se pueden ver gran variedad de estatutos legales que surgen tratando de regular y proteger la propiedad intelectual, como lo que se busca desarrollar más en Colombia con la firma del TLC con Estados Unidos.

El conocimiento y todo aquello que surge del capital intelectual que posee una empresa busca ser usado para un mejor desarrollo y administración de está, la administración ha evolucionado mucho en términos de organización y formulación de estrategias, ya que el mundo comercial es cambiante y cada vez más competitivo, la necesidad de buscar la mejor manera de desempeñarse en él, es organizando estrategias flexibles que permitan a la organización sobresalir y cumplir con sus objetivos.

La contabilidad juega en esto un papel muy importante ya que necesita brindar información confiable y que sirva para el desarrollo de esas estrategias; hoy en día se puede observar que la contabilidad creada para mostrar valores financieros diseñados en una era industrial no da mayor trascendencia a lo que se necesita actualmente con la creación y administración del conocimiento. El verdadero valor a mostrar para los accionistas e inversionistas de una empresa no es la rentabilidad a corto plazo que esta pueda generar sino la sostenibilidad y competitividad de ese negocio en el mercado (Dìaz Inchicaque, 2003). Es por eso que se han creado y se hace tanto énfasis en los diferentes métodos u medidas diseñados para medir el desempeño y crear una mayor seguridad a los dueños de las compañías. El capital del conocimiento es una nueva herramienta a trabajar que requiere de mayor importancia por parte de los contadores para generar una información más confiable y acertada a la toma de decisiones en los diferentes niveles de toma de decisión, ya sea nivel estratégico, táctico u operativo. 


\section{Capital Intelectual}

Nace a raíz de la existencia de activos inmateriales que afectan a la empresa en su valor en el mercado y que muestra diferencia en el valor reflejado por la contabilidad en libros de la empresa.

Se puede entender como "el conjunto de sistemas/procesos conformado por el capital humano, el capital estructural y el capital relacional, orientados a la producción y participación de conocimiento en función de objetivos estratégicos" (mantilla, 2004).

En el capital intelectual encontramos aquellos llamados activos intangibles los cuales hace referencia el Decreto 2649/93 en el Art. 66 (en Colombia) y la IAS 38 (aprobada por el IASC) en donde define a estos como aquel activo identificable, de carácter no monetario y sin apariencia física, que se tiene para producir bienes y servicios, para ser arrendado a terceros o para funciones administrativas del ente (Blanco Luna, 2002). También se encuentran el conocimiento individual como tal que debe ser convertido en información o conocimiento de uso para la organización, el cual se hace más difícil de calcular al no poder ser medido de una forma mas objetiva como tal.

Así entonces, según Mantilla (2004) los elementos que conforman el capital intelectual son:

\section{Capital humano}

- Saber cómo

- Educación

- Calificación vocacional 
- Conocimiento relacionado con el trabajo

- Valoración ocupacional

- Valoración sicométrica

- Competencias relacionadas con el trabajo

- Espíritu emprendedor, capacidad de innovar, habilidades proactivas y reactivas, capacidad de cambiar.

\section{Capital estructural (organizacional)}

2.1 Propiedad Intelectual

- Patentes

- Copyright

- Derechos de diseño

- Secretos comerciales

- Marcas registradas

- Marcas de servicio

2.2 Activos Infraestructurales

- Filosofía de la administración

- Cultura corporativa

- Procesos gerenciales

- Sistemas de información

- Sistemas de trabajo en red

- Relaciones financieras

\section{Capital relacional (del cliente)}

- Marcas 
- Clientes

- Lealtad del cliente

- Nombre de la compañía

- Órdenes en espera

- Canales de distribución

- Colaboración de negocios

- Acuerdos de licencias

- Contratos favorables

- Acuerdos de franquicias

- Rentabilidad por el cliente

- Rentabilidad por el producto

Para medir el capital intelectual existe, como ya se vio anteriormente, muchas alternativas, el dilema radica en que estos no cuentan con una suficiente objetividad donde refleje de manera razonable el desempeño del conocimiento en la organización.

Colombia y en general los países Latinoamericanos, con la apertura global de mercados y el proceso de firma del TLC, están viviendo cambios en muchos de los ámbitos del desarrollo nacional; desde un aspecto contable, en cuanto la implementación de normas que en casos como la contabilidad financiera, o lo que se utilizará; contabilidad del conocimiento, difieren de lo estipulado con la normativa nacional (Como el DR. 2649/93 en Colombia que se rige mas por lo fiscal); así también cambios en cuanto a la medición, uso y generación de conocimiento que se ve afectado de manera positiva o negativa con la firma del TLC. 


\section{La propiedad intelectual}

Del capital intelectual, el tema con mayor relevancia a tratar con respecto a lo que se vive en el TLC es la propiedad intelectual, un punto de mucho interés por discutir y en el que se encuentran factores en contra y a favor como tratamos anteriormente, pero para conocer un poco más a lo que esto se refiere le daremos un fin con una profundización en la definición de este concepto.

La propiedad intelectual "tiene que ver con las creaciones de la mente" ${ }^{7}$. Se divide en dos categorías:

- La propiedad industrial, que incluye las invenciones, patentes, marcas, dibujos y modelos industriales e indicaciones geográficas de origen; y

- El derecho de autor, que abarca las obras literarias y artísticas, tales como novelas, poemas y obras de teatro, películas, obras musicales, obras de arte, tales como dibujos, pinturas, fotografías y esculturas, y diseños arquitectónicos.

Estos componentes de la propiedad intelectual y sobre todo lo que respecta a los derechos de autor son tratados con mucho interés por las leyes y autoridades colombianas aunque no con mucho éxito; aunque en el país se hace énfasis en lo dañino que es no respetar esto derechos y apoyar la piratería, es de los delitos más comúnmente cometidos en Colombia. Es por razones como estas que en Colombia la cultura muchas veces no prospera más de lo que debería, no es que no se encuentre talento o mentes brillantes en el país, si no que el apoyo para desarrollar capital intelectual es muy pobre y se escapa inclusive muchas veces en busca de protección y soporte a otros países.

${ }^{7}$ OMPI. Organización Mundial de la Propiedad Intelectual. En: http://www.wipo.int/about-ip/es/ 
Colombia ha firmado acuerdos, por ejemplo con la Comunidad Andina (CAN) y se espera firmará otro con Estados Unidos en el TLC, donde ratifica su interés por la protección de la propiedad intelectual a lo que hace referencia: "Las Políticas sobre Propiedad Intelectual tienen por objeto garantizar, a nivel hemisférico, la creación de un sistema de protección sólido y dinámico de apoyo e impulso a la innovación tecnológica y la creatividad artística. Esto traduciría a un incremento en la realización de inversiones y transferencia de tecnología en una mayor cantidad de sectores productivos, así como en un entorno adecuado, simultáneamente permitiendo promover los Intereses de Colombia", sin embargo aunque se busque este objetivo suena un poco utópico el conseguirlo, ya que en Colombia no se ve realmente con fuerza la creación de innovación. Por más que se esté en la búsqueda de una mejor y más estable normativa aún hace falta mucho compromiso por el verdadero cumplimiento del objetivo de ésta.

\section{Conclusiones}

Teniendo una reseña de lo que es el concepto en si de capital intelectual, propiedad intelectual y de cómo los contadores jugamos un papel importante en su gestión, así como también del efecto que tendremos que vivir no sólo como colombianos si no como profesionales responsables en un campo con la firma del TLC podemos concluir:

1 Colombia es un país en vía de desarrollo, que ha entrado en su segunda etapa hacia la globalización y adaptación de ciertos estándares y normas que para nuestro estudio se refieren a la Propiedad Intelectual,; ésta situación nos refleja como Colombia se desarrolla en este entorno de una manera poco competitiva frente a economías como la Estadounidense la

\footnotetext{
${ }^{8}$ Propiedad intelectual, en: http://www.mincomercio.gov.co/VbeContent/NewsDetail.asp?ID=211\&IDCompany=4
} 
cual cuenta con grandes diferencias en cuanto al tamaño, la infraestructura física, la abismal diferencia en términos de uso y propiedad de los avances tecnológicos; y a partir de este racionamiento podemos preguntarnos ¿Cómo se puede pretender que con el TLC haya un desarrollo económico y un mejoramiento de la calidad de vida a partir de mejores productos, si existen grandes diferencias entre la economía de los EE.UU. y la Colombiana?. Esta pregunta se ve enmarcada por múltiples soluciones, y la ves por la contradicción de la acción del gobierno quien con sus "buenas intenciones" de expandir el desarrollo de los mercados, o al menos eso es lo que promulga el TLC, esta permitiendo que el gobierno de los EE.UU. interfiera y controle de cierta manera, los recursos nacionales de los que privilegiadamente gozamos.

2 El TLC en uno de sus ideales ilusorios buscar enfocarse en la premisa referente la propiedad intelectual, la cual indica que el acceso a los bienes públicos globales, tales como medicamentos, alimentos y seguridad, ofreciendo estos de una manera óptima y equitativa desarrollándose así gracias a las tecnologías y el conocimiento; reconocemos que esta premisa es acertada pero incierta y poco fehaciente en la práctica, como se refleja en el caso colombiano, ya que además de las dificultades sociales que estamos viviendo en el sector de la salud, y que podrían verse afectadas gravemente con las medidas pactadas en el TLC, de la siguiente manera:

a. Medicamentos: la accesibilidad a nuevos medicamentos y avances tecnológicos en salud, nos vemos afectados económicamente en primera medida gracias a acuerdos internacional sobre propiedad intelectual como los ADPIC, y ahora inmersos en el TLC, los colombianos tendríamos que asumir costos adicionales para el tratamiento de ciertas enfermedades; por otro lado cabe reconocer que 
dicha "apertura económica" al mercado estadounidense, nos podría representar mayor acceso a nuevos medicamentos, pero ¿a que precio? Esa es la gran incógnita puesto que no es tan fácil cumplir con esto, debido a las abismales diferencias entre estas naciones relacionadas.

b. Patentes: En Colombia la vigencia de las patentes de invención es de 20 años y de 10 años para las patentes de modelo de utilidad, pero centrándonos en las primeras, el interés norteamericano se ve enmarcado por el ánimo de aumentar dicho tiempo, por medio de tácticas como el hecho de compensar retrasos injustificados que ocurran en la aprobación de la patente, representada esta como una protección adicional, pero el problema esta en que conocemos la intención de EE.UU. con estas propuestas, más no se ha precisado al público el plazo final pactado para este tipo de casos, pero al querer extenderlo se busca aumentar el beneficio económico de grandes empresarios, perjudicando a personas que de alguna manera obtienen beneficios económicos con la reproducción de la información. Sin embargo específicamente en el caso de los productos farmacéuticos y el proceso para su aceptación y circulación en el mercado, al aumentar el tiempo de la patente por retrasos irrazonables se favorecen las gigantescas corporaciones como DuPont de Estados Unidos, Bayer de Alemania, Monsanto de Estados Unidos, Sygenta de Suiza, Dow de Estados Unidos y Basf de Alemania, las cuales controlan el 70\% del mercado farmacéutico, y como vemos serían las que lo controlarían dejando de la lado la labor de pequeñas farmacias. Frente a este tema es de importancia ejemplificar la intención provechosa de EE.UU., en su oposición a que las patentes basadas en material genético extranjero tengan que ser avalados por el país de origen de dicho material, puesto que sus esfuerzos se fijan hacia la eliminación de barreras legales que 
en Colombia les impiden patentar animales y plantas, por ejemplo, lo cual a su vez nos afecta en cuanto a la conservación de nuestra biodiversidad, representando un peligro a culturas aborígenes aún existentes en el país.

3. Colombia es un país rico en biodiversidad y en multipluralidad de razas y conocimientos que podrían llegar a no ser respetados y desnacionalizados si se permite el uso de estos a extranjeros de una manera similar que a los nacionales, este también es un tema que afecta no solo a la propiedad intelectual como tal sino a nuestro país en general en el progreso y formación de nuestra cultura, si ya se puede observarse como la cultura extranjera ha influenciado muchas de las costumbres del diario vivir nuestro, con el TLC se verá más marcado este efecto, el problema no es aprender de otras culturas y conocer de ellas, el problema es que no respeten nuestra propia cultura y se busque influenciar en ella, nuestros recursos son dados en nuestro territorio nacional para ser aprovechados en nuestro crecimiento económico, así que el dejar que otros países utilicen estos en búsqueda de un "bien común" sin restricciones y con igual trato a los habitantes nacionales no parece una manera lógica de solidificar y ayudar a la economía del país, por que está muy clara la magistral ventaja que nos lleva en tecnología EE.UU., que con esta podrían usar mucho mejor nuestros recursos que nosotros quienes somos un país en vía de desarrollo y con mucho por aprender sobre todo en la utilización y valoración del capital que tenemos, sea en Recursos Naturales o en generación de conocimiento y tecnología.

4. Colombia es un país con grandes capacidades de progreso, y posee personajes así como empresas que pueden demostrar esto; pero aun 
así en el campo contable falta mucho por avanzar y aprender, la contabilidad esta cambiando y los contadores deben saber desempeñarse mucho más allá del área fiscal y tributaria, la contabilidad se ha convertido en una herramienta clave que trasciende las creencias de la simple teneduría de libros y de la obligación legal; la Contabilidad del Conocimiento es un claro ejemplo de esto, la necesidad se ha acrecentado mucho más y la vital reacción al cambio se ve reflejado desde el punto de vista de la generación de capital intelectual y de la necesidad de administrar y valora este, en algo tan importante como es la propiedad intelectual y lo que la firma del Tratado de Libre Comercio influirá, incumbe a todos los contadores los cuales están siendo considerados más convenientes como instrumentos de información para la gerencia del capital intelectual humano, y para el manejo de esa parte de la empresa que aun se tiene desconcierto del como se debe administrar; el capital intelectual afecta el valor de la empresa en el mercado, en el desempeño y curso de está, así que es importante no llegar a subestimar el valor real de la empresa, pero también saber demostrar el progreso y las fortalezas que ha esta le traen el poseer Activos que sobrepasan los limites de lo legal.

5. Un factor importante a tener en cuenta es el de no caer en el error de cosificar al ser humano que si bien es el generador del conocimiento, también posee características que son necesarias respetar, he ahí uno de los dilemas de la valoración del conocimiento, que es desarrollado por personas que trasmiten éste de manera que involucran no solo conceptos, sino también sentimientos y demás cualidades personales que poseen, que serian imposible poder llegar a darles un valor comercial. Se puede afirmar que el conocimiento tiene una ventaja que 
hace que siga aumentando su valor en la organización, y es que al no ser absoluto sigue en un constante surgimiento.

\section{Bibliografía}

Blanco Luna, Yanel (2002). Normas nacionales e internacionales de contabilidad. IAS 38 Activos Intangibles. Bogota D.C.

Díaz Inchicaqui, Miguel. La contabilidad y la gestión del conocimiento. En: sisbib.unmsm.edu.pe, consultada en octubre de 2006.

Gomez Carvajal, Viviana María (2005). Escuela Interamericana de Bibliotecología. Medellín: Universidad de Antioquia.

González, Sara y Mascareñas, Juan. Universidad Complutense de Madrid. "La Globalización de los Mercados Financieros". En: www.ucm.es/info/jmas/temas/global.pdf\#search=\%22globalizacion\%20mercados\%22, consultada en octubre de 2006.

Life style Extra: Finance, news \& Entertainment. En: www.Ise.co.uk/financeglossary.asp, consultada en octubre de 2006.

Madrid, Luis Ángel. "La salud pública y la discusión de Propiedad Intelectual en el TLC Colombia-Estados Unidos". En: www. usergioarboleda.edu.co/tlc/\#*.htm, consultada en octubre de 2006.

Mantilla, Samuel Alberto (2004). Capital intelectual \& Contabilidad del Conocimiento. Bogotá D.C.: ECOE Ediciones.

OMPI. Organización Mundial de la Propiedad Intelectual. www.wipo.int., consultada en octubre de 2006.

Organización Mundial de la Propiedad Intelectual,. En: www.wipo.int/portal/index.html.es, consultada en octubre de 2006.

Organización Panamericana de la Salud. ¿EI TLC tiene impacto en la salud de los colombianos?". En: www.col.ops-oms.org, consultada en octubre de 2006.

Parker Rosell, Héctor. Economía del conocimiento. En: Planeacion.cicese.mx., consultada en octubre de 2006. 
Skyrme, David. Knowledge Management. En:

www.skyrme.com/insights/22km.htm, consultada en octubre de 2006.

Stewart, Bennet. The real key to creating wealth. En: www.eva.com, consultada en octubre de 2006.

www.mincomercio.gov.co, consultada en octubre de 2006.

www.usergioarboleda.edu.co/tlc/propiedad intelectual tlc mirada.htm, consultada en octubre de 2006.

www.proexport.xom.xo/VbeContent/Library/documents/DocNewsNo2726Documen tsNo5547.PDF, consultada en octubre de 2006.

www.elsignovital.com.co, consultada en octubre de 2006. 